\title{
Eriksen's two-phase model of spatial selective attention: Physiological evidence of trial-mixing-dependent response inhibition
}

\author{
J. Toby Mordkoff ${ }^{1} \cdot$ Peii Chen ${ }^{2,3}$ \\ Accepted: 13 September 2020 / Published online: 18 October 2020 \\ (C) The Psychonomic Society, Inc. 2020
}

\begin{abstract}
The early work of Charles W. Eriksen and colleagues provided us with both the flanker task and the concepts of response competition and continuous flow. The model of the flanker task that Eriksen and colleagues developed also includes the idea that processing occurs in two phases and the specific claim that pro-active response inhibition is employed to prevent errors under certain conditions. We first replicated and extended the behavioral evidence that motivated this specific claim and then tested it using a variety of physiological measures. We verified the prediction of Eriksen's Two-Phase Model of Spatial Selective Attention using the lateralized readiness potential and contingent negative variation. We also clarified a detail of the model using electromyographic activity and response force. We note that this contribution of Charles W. Eriksen has not received the attention that it deserves and that several recent models might need to be revised in light of Eriksen's work.
\end{abstract}

Keywords Cognitive and attentional control $\cdot$ Attention: selective $\cdot$ Attention and executive control

\section{Introduction}

The history of research and theorizing on human information processing is marked by a series of rapid advances, such as when a useful new task is devised or an insightful new model is developed. The work of Charles W. Eriksen and students and colleagues provides several examples of such advances. In his best-known line of work, Eriksen provided us with what has become one of the most popular paradigms in cognitive psychology - the flanker task - along with the concepts of continuous flow of information between mechanisms (as an alternative to discrete stages) and competition between alternative responses. When Eriksen's early work is cited, it is usually for one of these reasons. But another innovation of this work is the idea that processing may proceed in two phases and the specific claim that response activation is sometimes inhibited during the first phase in order to prevent errors. This last idea is seldom mentioned, but is the focus of the

J. Toby Mordkoff

jonathan-mordkoff@uiowa.edu

1 Department of Psychological and Brain Sciences, G60 PBSB, University of Iowa, Iowa City, IA 52242, USA

2 Kessler Foundation, West Orange, NJ, USA

3 Rutgers New Jersey Medical School, Newark, NJ, USA current paper. It is introduced and explained in detail by describing the specific model of the flanker task that was developed by Charles W. Eriksen and colleagues. ${ }^{1}$

\section{The flanker task and Eriksen's two-phase model of spatial selective attention}

The flanker task was devised and refined by Eriksen and colleagues (starting with Eriksen \& Hoffman, 1972, 1973) as a method of studying spatial selective attention or, conversely, as a way of exploring the effects of irrelevant stimuli when the location of the target is known in advance. The name for the task comes from the fact that the irrelevant stimuli flank the target within the display. In a typical example, the task is twoalternative forced-choice with two different targets assigned to each of the responses - for example, the letters $\boldsymbol{C}$ and $\boldsymbol{S}$ assigned to one response and the letters $\boldsymbol{H}$ and $\boldsymbol{K}$ assigned to the other response. The target appears at fixation (or directly above), flanked on each side by equal numbers of irrelevant "noise" letters. In some experiments the eccentricity of the flankers is fixed; in other experiments the spatial separation between the flankers and target is varied. Most of all: the

\footnotetext{
${ }^{1}$ If the reader is interested in quickly tracing the evolution of Eriksen's model, the authors suggest the following five papers (to be read in chronological order): Eriksen and Hoffman (1973); Eriksen and Eriksen (1974); Eriksen and Schultz (1979); Coles, Gratton, Bashore, Eriksen, and Donchin (1985); Gratton, Coles, Sirevaag, Eriksen, and Donchin (1988).
} 
relationship between the flankers and the target is systematically varied. The flankers can be: (1) the same letter as the target and, therefore, associated with the same response as the target (congruent - identical; e.g., C C C ); (2) a different letter assigned to the same response as the target (congruent - alternative target; e.g., S C S ); (3) visually similar to the target, but not actually assigned to either response (neutral visually similar to congruent.; e.g., G C G ); (4) visually similar to targets assigned to the opposite response (neutral - visually similar to incongruent; e.g., N C N ); or (5) one of the letters assigned to the opposite response from the target (incongruent; e.g., H C H ). In some experiments, a control condition is also included - i.e., the target (alone) without any flankers. The trials in the control condition may be randomly inter-mixed with the trials that include flankers (mixed control) or run a separate block (blocked control).

A revised plot of the results from the most famous such experiment (Eriksen \& Eriksen, 1974) is provided in Fig. 1. There are several things worth noting about these data. First, when the flankers are presented quite close to the target and, therefore, close to fixation $\left(.06^{\circ}\right.$ spacing), they produce a congruence effect that depends on their name. In this case, if the flankers are letters that are assigned to the same response as the target (congruent), mean response time (mRT) is lowest; if the flankers are letters assigned to the opposite response (incongruent), $\mathrm{mRT}$ is highest; if the flankers are letters that are not assigned to either response (neutral), $\mathrm{mRT}$ is intermediate. Second, when the flankers are presented much farther from fixation $\left(1^{\circ}\right.$ spacing), their effect depends on their general appearance, instead of their name. Assuming that the target is the letter $\boldsymbol{C}$, for example, the three conditions with curvedline flankers (identical (i.e., C C C), alternative target (i.e., S C S), and visually similar to congruent (e.g., G C G)) produce equal mRTs that are all lower than the two conditions with

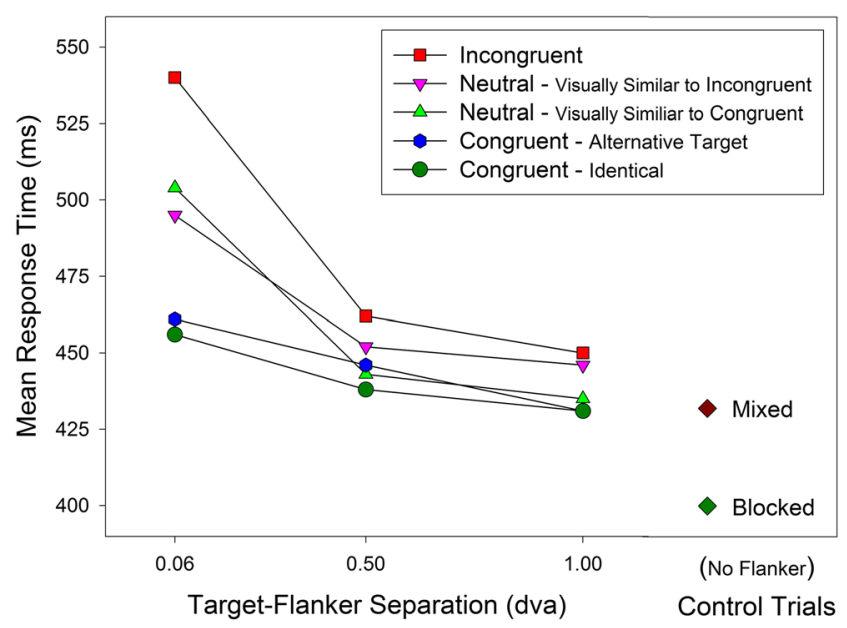

Fig. 1 Revised and updated copy of Fig. 1 from B. A. Eriksen and C. W. Eriksen, 1974, Perception \& Psychophysics, Vol. 16, No. 1, pp. 143-149; modern labels for the five experimental conditions have been added for convenience angular flankers (incongruent (e.g., $\mathbf{H ~} \mathbf{C} \mathbf{H}$ or $\mathbf{K} \mathbf{C ~} \mathbf{K}$ ) and visually similar to incongruent (e.g., N C N)). Finally, note that trials without any flankers (i.e., control trials) produce a lower mRT when run in a separate block than when mixed with the trials that do include flankers.

These results led Eriksen and colleagues to propose a specific model of performance of the flanker task: the Two-Phase Model of Spatial Selective Attention (see footnote 1; also summarized in Gratton, Coles, \& Donchin, 1992). The TwoPhase Model incorporates elements from a variety of sources, from vision science to cognitive control, and has stood the test of time very well. At its core, the model is the archetype of continuous flow in that information is always being passed "forward" freely, from stimulus encoding to response activation. The model also includes competition between alternative responses and embodies the claim that processing in the flanker task occurs in two distinct phases.

The first phase of processing is non-selective. During this time, all items within the display receive equal processing, subject to acuity limits (and, maybe, lateral masking). Items close to fixation will be fully identified, such that the names of these letters will be available to subsequent processes. Items far from fixation will only be classified in terms of their general appearance, such as curved versus angular for $\boldsymbol{C}$ and $\boldsymbol{H}$, respectively. Thus, flankers far from fixation would partially activate the names of all letters that they resemble, such that a $\boldsymbol{G}$, for example, would provide some activation for both $\boldsymbol{C}$ and $\boldsymbol{S}$, but not for either $\boldsymbol{H}$ or $\boldsymbol{K}$. In either case, letter-name information is continuously transmitted to response-selection processes, such that any item that is even partially identified as having the name of a letter that is assigned to a response will prime that response. If multiple responses receive activation, they compete with each other via inhibitory cross-connections. The processing that occurs during the non-selective phase is what produces the flanker effect. This has been found to have notable consequences for subsequent trials (see, e.g., Botvinick, Braver, Barch, Carter, \& Cohen, 2001; Gratton et al., 1992) and is reflected in several distinct event-related brain potentials (see, e.g., Donohue, Appelbaum, McKay, \& Woldorff, 2016; Tillman \& Wiens, 2011; Zhou, Xiong, Cheng, \& Wang, 2019).

The second phase of processing is spatially selective. During this time, the target receives much more attention than any of the flankers. In the extreme, all processing of the flankers may cease. The processing that occurs during the selective phase is what allows the correct response to be produced on a vast majority of trials, even those on which the priming that occurs during the non-selective phase is mostly in favor of the incorrect response. The timing of the shift between the two phases is determined by the difficulty of locating and selecting the target. Wider spatial gaps between items, for example, allow the shift from non-selective to selective processing to occur more quickly. Computational modeling 
(starting with Cohen, Servan-Schreiber, \& McClelland, 1992) has been used to test whether this is a discrete transition or occurs gradually (see, e.g., Hübner, Steinhauser, \& Lehle, 2010; Liu, Holmes, \& Cohen, 2008; White, Ratcliff, \& Starns, 2011).

\section{Response inhibition and blocked versus mixed control trials}

The Two-Phase Model as described above does remarkably well in explaining the results from the five experimental conditions of the classic experiment (see left side of Fig. 1), but not the results from the control trials (see right side of Fig. 1). Importantly, the model includes one other component - response inhibition - that also depends on the phase of processing. This aspect of the model has often been ignored (or has been over-shadowed by other issues), even though some early descriptions of the model refer to the two phases in terms of this inhibition, instead of in terms of target selection. In order to avoid errors that are based on the identity or general appearance of the flankers, "an inhibitory process is required to prevent the responses from running off willy-nilly" (Eriksen \& Eriksen, 1974, p. 144). In other words, those mechanisms responsible for producing responses are suppressed proactively or in advance until the non-selective phase of processing is done. The modern label for this is "pro-active inhibition" (see, e.g., Braver, 2012.)

In contrast to the processes responsible for response competition, this type of inhibitory control is not aimed at specific responses; instead, it acts to prevent any response from being made (prematurely). Thus, it more closely resembles the type of control that is thought to be used to perform the stop-signal task (see, e.g., Lappin \& Eriksen, 1966; Logan \& Cowan, 1984; Verbruggen \& Logan, 2008), where all types of motor output are simultaneously suppressed (see, also, Wessel \& Aron, 2017). The key evidence in support of this claim comes from the effect of blocking on $\mathrm{mRT}$ for control trials (see right side of Fig. 1). Response inhibition is only required when some trials within the block involve flankers that are associated with the incorrect response. "In the mixed control, ... when a subject initiated a trial it would be to his advantage to have inhibitory processes activated, whereas in the blocked condition, ... the subject could have discarded any inhibitory process in preparation for a trial" (Eriksen \& Eriksen, 1974, p. 148). Thus, processing on mixed control trials includes response inhibition, but processing on blocked control trials does not. The present work tested this specific claim using a variety of physiological measures, including event-related brain potentials (ERPs), electromyographic activity (EMG), and response force.

\section{Behavioral pilot experiment}

It is standard "good practice" in ERP research to reduce the likelihood and influence of various artefacts (see, e.g., Luck, 2005). One source of such artefacts that exists in the original comparison between blocked and mixed control (Eriksen \& Eriksen, 1974) is the variability in the number of stimuli presented across the trials within a block. In blocked control, all displays within the block contain exactly one letter (the target); in mixed control, some displays contain only one letter (i.e., the target in a control trial), while other displays contain many letters (the target plus the flankers in congruent, incongruent, and neutral trials). Note, however, that the claim that trial-type mixing is what determines whether responses are inhibited during the non-selective phase of processing should also apply to trials with neutral flankers. Thus, blocked neutral should also produce faster responses than mixed neutral, because only the latter would involve inhibition. The main goal of the behavioral pilot of the present study was to verify this simple prediction.

The second goal of the behavioral pilot was to replicate the block/mixed effect (on neutral trials) in the simplest possible task. This was done to test the conditions that were planned for the main physiological experiment. In contrast to the original blocked/mixed experiment (Eriksen \& Eriksen, 1974), which involved two different targets per response and six different flanker-target relationships (see Fig. 1), the present experiment used only one target per response and included only three conditions: congruent - identical, incongruent, and neutral. (Hereafter, congruent-identical is referred to as simply congruent.) Similar to the switch from control trials to neutral trials for the critical data, there is no reason to believe that the other three flanker-target conditions are needed to produce the blocked/mixed effect. The present experiment also used only one value of flanker-target separation, instead of three values, and the stimuli were much larger than those used by Eriksen and Eriksen (1974), in order to avoid the issue of visual similarity.

Finally, instead of simple (on/off) buttons or a left/right joystick (as used in the original), the pilot experiment used zero-displacement, force-sensitive keys. This was also done to match the planned method for the main experiment, which would include measures of both response force and electromyographic activity (EMG). EMG is best measured when muscles tense without causing any actual movement.

\section{Participants}

Twenty participants were recruited from an Elementary Psychology subject pool and each was run in a single, 1-h session. All participants reported normal or corrected-tonormal vision and no motor deficits. All participants provided 
informed consent and all procedures were approved by the Institutional Review Board.

\section{Stimuli and response apparatus}

All stimuli were presented in white on a black screen. The targets were the letters $\boldsymbol{H}$ and $\boldsymbol{S}$, assigned to the left and right index fingers by odd/even counterbalancing. The neutral flanker was the letter $\boldsymbol{O}$. There were two copies of the flanker of each side of the target. Thus, example displays would be $\mathbf{H}$ H H H H (congruent), S S H S S (incongruent), and O O H O $\mathbf{O}$ (neutral). From the standard viewing distance of $65 \mathrm{~cm}$, each letter was $1.15 \times 0.80$ degrees of visual angle (dva) and the center-to-center separation between letters was 1.85 dva. The fixation cross was 0.35 dva square.

The participants responded by pressing firm levers that were attached to piezo-electric force transducers (PCB Piezotronics; Depew, NY, USA). The criterion for registering a response was set to $100 \mathrm{cN}$, which is approximately the "weight" of $100 \mathrm{~g}$ at sea level. The onset of the response was then determined by back-tracing along the force-time function to the last point in time at which force was greater than $10 \mathrm{cN}$ or the first local minimum was found. In other words, response choice used a criterion of $100 \mathrm{cN}$, but response time used a criterion of $10 \mathrm{cN}$.

\section{Design and procedure}

After providing consent, the participants were given written instructions that explained the task, including the targets-tokeys mapping, and stressed accuracy over response time (RT). The participants then completed two short (20-trial) blocks of practice, followed by 12 full blocks of approximately 40 trials. In the full blocks, there were 36 planned trials; for the mixedconditions blocks, there were 12 trials in each of the three conditions; for the neutral-only blocks, all 36 trials involved the neutral flanker. The trials in each condition were evenly divided with regard to target letter (and, therefore, which response was correct). Prior to the 36 planned trials in a block, there were three randomly selected "warm-up" trials. After an error, there was a randomly selected "recovery" trial. All warm-up and recovery trials were excluded from the analysis.

For all participants, the first block of practice was in the neutral-only condition, while the second block of practice was mixed conditions. During practice, participants were given detailed feedback after every error, which included a reminder not to tap the response keys (which was defined as an increase of $50 \mathrm{cN}$ or more in a $2-\mathrm{ms}$ period) and to avoid pressing much harder than necessary (which was defined as a peak force over $1,000 \mathrm{cN}$ ). The practice data were excluded from all analyses.

The 12 full blocks were divided into three sets of four, with three of each four being mixed conditions and one of each four being neutral only. This was done to equalize the amount of data for blocked and mixed neutral. The position of the neutral-only block within each set of four was counterbalanced across participants. Only the second and third sets of four blocks were retained for analysis. In other words, the first set of four full blocks was also treated as practice.

Each trial began with the presentation of the fixation cross for $350 \mathrm{~ms}$. This was followed by a blank screen for $150 \mathrm{~ms}$. The final display with the target and flankers was then shown until a response was detected or 1,500 ms had elapsed (at which point the trial was treated as an omission error). The inter-trial interval was $2,000 \mathrm{~ms}$.

\section{Results and discussion}

The behavioral pilot was highly successful. The blocked/ mixed effect on neutral trials was $21.92 \pm 5.04 \mathrm{~ms} ; \boldsymbol{t}(19)=$ $4.35, p<.001$, adj $\widehat{\eta}_{\mathrm{p}}^{2}=.473 .^{2}$ Mean RT for blocked neutral was 425.01 ms with a mean error-rate of $1.27 \%$; mean RT for mixed neutral was 446.93 ms with $1.89 \%$ errors. While not relevant to present purposes, in the mixed blocks, the flanker congruency effect was $40.79 \pm 4.73 \mathrm{~ms} ; \boldsymbol{t}(19)=8.62, p<.001$, adj $\widehat{\eta}_{\mathrm{p}}^{2}=.786$. Mean RT for congruent was $441.27 \mathrm{~ms}$ with $0.73 \%$ errors; mean RT for incongruent was $482.08 \mathrm{~ms}$ with $2.98 \%$ errors. In short, the behavioral pilot succeeded in extending the findings of Eriksen and Eriksen (1974) to include blocked versus mixed neutral trials using modern equipment and a much simpler design.

\section{Main physiological experiment}

The specific claim to be tested is that the advantage of blocked over mixed neutral trials is due to the absence of response inhibition in the former condition (Eriksen \& Eriksen, 1974). The present test was conducted using several physiological measures, including the lateralized readiness potential (LRP), contingent negative variation (CNV), electromyographic activity (EMG), and response force. (For a detailed introduction to the logic and method being employed, see Danek \& Mordkoff, 2011; Mordkoff \& Grosjean, 2001). Eriksen's Two-Phase Model predicts a larger LRP for blocked over mixed neutral trials, because the LRP is an index of response preparation, which would be suppressed by response inhibition. At the same time, the model does not predict differences in the CNV, because the model does not posit general (non-motoric) changes in either arousal or trial preparation.

\footnotetext{
${ }^{2}$ Effect size is reported as an adjusted partial-eta-squared because the unadjusted value (produced by most statistical packages) suffers from noticeable positive bias, especially when the true effect size or sample is small; see Mordkoff, 2019. Note that values of adj $\widehat{\eta}_{\mathrm{p}}^{2}$ can be negative when the true effect is very small or non-existent and should not be rounded up to zero; see Okada (2017).
} 
With respect to EMG and response force, the Two-Phase Model, as originally presented, does not make clear predictions. However, these data can be used to discriminate between different sub-types of the model. If, for example, one takes seriously the suggested parallel between stop-signal processing and the response inhibition that is claimed to occur when incongruent trials are mixed with the neutral trials, then one would predict no effect of trial-type mixing on either EMG amplitude or response force. This holds because responses, once initiated, are unaffected by any of the specifics of pre-motor processing (see, e.g., Logan \& Cowan, 1984; Osman, Kornblum, \& Meyer, 1986). In contrast, if the concept of continuous flow applies within the motor system (in addition to between perceptual and central processes), then one would predict differences in EMG and response force that match the differences observed in the LRP.

The lateralized readiness potential This event-related brain potential (ERP) is an on-line measure of response preparation (for an introduction, see, e.g., Coles, 1989). It is defined as the mean of the difference between electrode sites $\mathrm{C}^{\prime}{ }^{\prime}$ and $\mathrm{C}^{\prime}$ ' on left-hand trials and the difference between $\mathrm{C}^{\prime}{ }^{\prime}$ and $\mathrm{C}^{\prime}$ on right-hand trials (such that the value is positive). A parallel analysis is usually conducted using the left and right outer canthi in the places of $\mathrm{C}^{\prime}$ and $\mathrm{C}^{\prime}$, respectively, such that an artefact plot can be included in the same figure. The overall magnitude of the LRP in a given condition can be quantified as the mean during the $100-\mathrm{ms}$ interval immediately prior to response onset. The reason for only examining the LRP before the onset of a response is that sensory feedback, once a response has begun, will also affect the same electrode sites. Most of all, if response selection or preparation is being inhibited, then the amplitude of the LRP should be reduced. In contrast, if the threshold for activating a response is increased, then the amplitude of the LRP during the 100-ms interval immediately prior to response onset might be increased, instead (see, e.g., Danek \& Mordkoff, 2011).

Contingent negative variation $\mathrm{The} \mathrm{CNV}$ is a measure of general readiness, as distinct from preparation to make a specific response (for an introduction, see, e.g., Rockstroh, Elbert, Canavan, Lutzenberger, \& Birbaumer, 1989). It is measured at electrode site $\mathrm{Cz}$ immediately prior to the appearance of a predictable stimulus that will require a response. Note that the reaction stimulus must have a predictable onset, such as when it appears at a fixed delay after a warning. (In the present work, the target and flankers always appeared exactly $500 \mathrm{~ms}$ after the onset of the fixation cross.) The magnitude of the CNV can be quantified as the mean amplitude in the 100-ms interval immediately prior to stimulus onset.

EMG and response force These are highly correlated measures of response production (see, e.g., Gratton et al., 1988). When responses are made using the tips of the index fingers, EMG is measured on the forearms, over the bodies of the flexor digitorum profundus muscles. The signal is rectified before averaging. When used in close conjunction with other measures that each employ a 100-ms window (e.g., the LRP and $\mathrm{CNV}$ ), both EMG and response force can be quantified as the mean amplitude during the first $100 \mathrm{~ms}$ after response onset. (In other situations, other definitions are used - e.g., peak force; Giray \& Ulrich, 1993; Mordkoff \& Grosjean, 2001.)

\section{Participants}

A total of 22 participants were tested, the planned 20 plus two replacements for those lost to faulty equipment (a broken wire in one of the electrode caps). The participants were recruited by poster and paid US $\$ 20$ for completing two sessions at about the same time on consecutive days. All reported normal or correct-to-normal vision, no motor deficits, and no psychiatric conditions. All provided informed consent and the procedures were approved by the Institutional Review Board.

\section{General method}

During the first session, the participant performed the exact same experiment as the behavioral pilot (above), including the same written instructions and two practice blocks with detailed feedback. All of these data were excluded from analysis. During the second session, the participant performed the same experiment again, this time with new instructions that discouraged blinking or any eye or body movements between fixation onset and the end of a trial. During the two practice blocks, the experimenter informed the participant (via intercom) if a blink or eye-movement was detected, based on the electrooculogram (EOG) recordings, prior to any force production. As for the behavioral pilot, only the data from the last two sets of four blocks were retained for analysis.

\section{Physiological recording}

ERPs were recorded using caps with $\mathrm{Ag}-\mathrm{AgCl}$ electrodes (Electro-Cap International, Inc; Eaton, OH, USA) and an isolated, bio-electric amplifier (SA Instrumentation; San Diego, CA, USA). The critical electrode sites were $\mathrm{C}^{\prime}$ and $\mathrm{C} 4^{\prime}(1 \mathrm{~cm}$ anterior and superior to $\mathrm{C} 3$ and $\mathrm{C} 4$, respectively) and $\mathrm{Cz}$. Electrodes were also placed lateral to each outer canthi, below the right eye, at the mastoids, and on the forearms over the body of the index finger flexor muscle. The impedance of all EEG electrodes was below $5 \mathrm{k} \Omega$ and these signals were amplified by 20,000 with a band-pass of 0.01 to $100 \mathrm{~Hz}$. The EOG electrodes were below $10 \mathrm{k} \Omega$, amplified by 5,000 , and used $0.1-100 \mathrm{~Hz}$ filtering. The EMG electrodes were also below $10 \mathrm{k} \Omega$, amplified by 10,000 , and used $0.3-100 \mathrm{~Hz}$. 
All data were sampled at $250 \mathrm{~Hz}$. The sampling epoch started $200 \mathrm{~ms}$ before the appearance of the fixation cross and continued until 1,000 ms after the target and flankers were shown.

\section{Artifact removal, participant loss, and data reduction}

The EOG data were first examined for blinks and horizontal eye-movements. Three participants were omitted from all further analysis for blinking on more than $25 \%$ of the trials. For the remaining participants, a mean of $13 \%$ of all trials were lost due to excessive EOG, blocking of a critical channel, or large amounts of drift. Next, a grand-average LRP, collapsing across all conditions in both types of block, was calculated for each participant. Six participants were omitted from all further analysis for exhibiting no measurable lateralization. (The loss of one-third of participants is typical of LRP studies.)

The data from the remaining 11 participants were then subjected to the planned set of three analyses. For the analysis and plots of the LRP, the data were locked to the onset of force production (i.e., $10 \mathrm{cN}$ ). The magnitude of the LRP in a given condition (i.e., blocked or mixed neutral) was quantified as the mean during the 100 -ms interval immediately prior to the onset of force production. For CNV, the data were locked to stimulus onset and the size of the CNV was defined as the mean during the 100 -ms interval immediately prior to the onset of final display. For EMG and response force, the data were locked to the onset of force production and the magnitudes were defined as the mean in 100-ms interval immediately following response onset.

\section{Results and discussion}

Mean response time The advantage of blocked over mixed neutral trials was again replicated. On the trials that were retained for analysis (i.e., those without blinks or other sources of artefact), the mean blocked/mixed effect was $28.42 \pm 4.10$ $\mathrm{ms} ; \boldsymbol{t}(10)=6.94, p<.001$, adj $\widehat{\eta}_{\mathrm{p}}^{2}=.811$. A parallel analysis of all trials showed a blocked/mixed effect of $27.47 \pm 5.13 \mathrm{~ms}$; $\boldsymbol{t}(10)=5.35, p<.001$, adj $\widehat{\eta}_{\mathrm{p}}^{2}=.715$. The mean flanker congruency effect on retained trials was $45.41 \pm 5.61 \mathrm{~ms} ; \boldsymbol{t}(10)=$ $8.09, p<.001$, adj $\widehat{\eta}_{\mathrm{p}}^{2}=.854$. In summary, not only was the blocked/mixed effect replicated, but there is no evidence that the addition of electrodes and the discouragement of blinks, or the removal of trials with potential artefacts had any noticeable influence.

Lateralized readiness potential The grand-average, responselocked LRPs are shown in Fig. 2, together with the horizontal EOG waveforms (which demonstrate no evidence of an eyemovement artefact prior to the onset of the response). As can be seen, the LRP for blocked neutral was larger than that for mixed neutral. During the $100-\mathrm{ms}$ interval just prior to

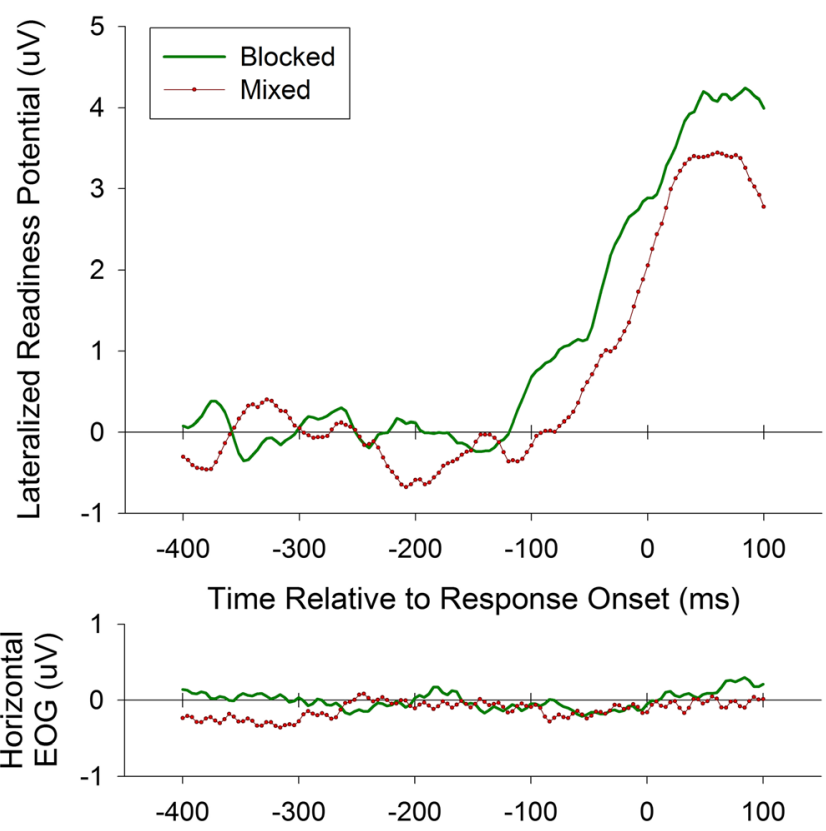

Fig. 2 Grand-average, response-locked lateralized readiness potentials (LRPs) for the blocked and mixed neutral conditions, and corresponding horizontal electro-oculograms (EOGs)

response onset, the advantage of blocked neutral was $0.92 \pm$ $0.32 \mathrm{uV} ; \boldsymbol{t}(10)=2.90, p=.016, \operatorname{adj} \widehat{\eta}_{\mathrm{p}}^{2}=.402$. This verifies the prediction from Eriksen's Two-Phase Model that response preparation is suppressed or inhibited in the mixed neutral condition.

Contingent negative variation The grand-average, stimuluslocked $\mathrm{Cz}$ waveforms are shown in Fig. 3, together with the vertical EOGs. The slow drift in vertical EOG appears to have been caused by some participants forcing themselves not to blink; in any event, the results for the CNV cannot be an artefact of differences in EOG, because the EOG waveforms are virtually identical. During the $100-\mathrm{ms}$ interval just prior to the appearance of the final display, the blocked/mixed effect was $0.04 \pm 0.33 \mathrm{uV} ; \boldsymbol{t}(10)=0.12, p=.907$, adj $\widehat{\eta}_{\mathrm{p}}^{2}=-.099$. Thus, the blocked/mixed effect on the CNV was negligible with no statistical significance. This rules out the idea that the blocked/mixed effect in mean RT is due to differences in overall arousal or other forms of non-specific trial preparation, as is claimed by Eriksen's model.

Motor measures The grand-average, response-locked EMGs and force curves are shown in Fig. 4. During the 100-ms interval just after the onset of the response, the blocked/ mixed effect on mean EMG was $-0.31 \pm 0.45 \mathrm{uV} ; \boldsymbol{t}(10)=$ $0.69, p=.506$, adj $\widehat{\eta}_{\mathrm{p}}^{2}=-.050$. During the same interval, the blocked/mixed effect on mean response force was $1.71 \pm 1.62$ $\mathrm{cN} ; \boldsymbol{t}(10)=1.05, p=.318, \operatorname{adj} \widehat{\eta}_{\mathrm{p}}^{2}=.009$. In short, there was no evidence that the blocked/mixed effect in mean RT was due to 

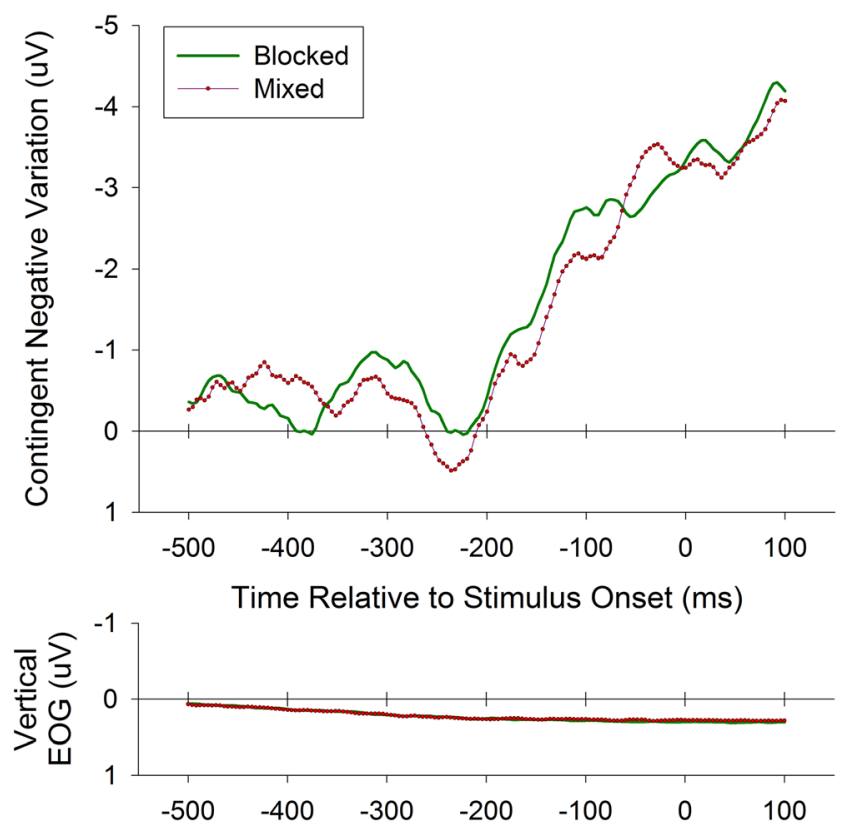

Fig. 3 Grand-average, stimulus-locked contingent negative variations (CNVs; voltages at electrode site $\mathrm{Cz}$ ) for the blocked and mixed neutral conditions, and corresponding vertical electrooculograms (EOGs) (note that time -500 is also fixation onset)

differences in response execution. Furthermore, these data provide some support for the idea that the response inhibition that depends on trial mixing in the flanker task (and produced
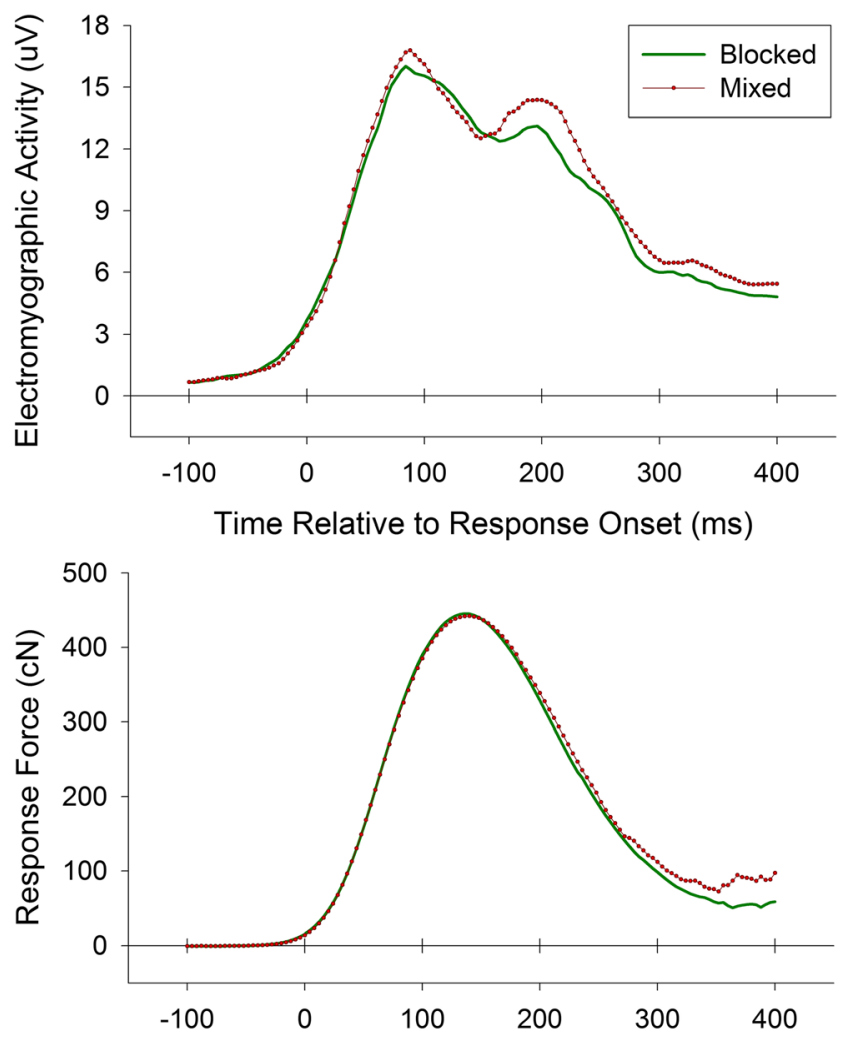

Fig. 4 Grand-average, response-locked electromyograms (EMGs) and amounts of response force for the blocked- and mixed-neutral conditions the observed difference in the LRP, above) is similar to that which is used to prevent responses in stop-signal tasks. In both cases, the inhibition only serves to either delay or prevent a response from being initiated; the actual execution of the response, if and when it occurs, is virtually unchanged. ${ }^{3}$

\section{Conclusion}

The early work of Charles W. Eriksen and colleagues provided us with one of the most useful and popular experimental paradigms - the flanker task (Eriksen \& Hoffman, 1972, 1973) and several concepts that are now central to our understanding of human information processing. These include the ideas that alternative responses compete for activation (Eriksen \& Eriksen, 1974) and that information flows continuously from one process to the next (Eriksen \& Schultz, 1979). But the model developed by Eriksen and colleagues also includes the specific claim that, under certain conditions, all responses are initially inhibited in order to prevent errors (Eriksen \& Eriksen, 1974). This claim was tested here using a variety of psychophysiological measures. Consistent with the idea that it is the inclusion of incongruent trials within a block that evokes the response inhibition, we observed smaller lateralized readiness potentials on mixed neutral trials than on blocked neutral trials. Consistent with the idea that it is response preparation in particular that is inhibited and not overall readiness or global arousal, we observed no differences in the contingent negative variation between mixed and blocked neutral trials. Finally, consistent with the suggestion that the inhibition that prevents premature errors in mixed blocks of the flanker task is similar to the inhibition that prevents responses in the stop-signal tasks, we observed no difference in either electromyographic activity or response force. Thus, we verified the predictions of Eriksen's Two-Phase Model of Spatial Selective Attention and reinforced the link between elements of the model and another form of inhibitory control.

Of course, verification of a model's prediction is not proof that the model is correct. There might be other ways to explain the difference in response preparation between blocked and mixed neutral trials without reference to changes in response inhibition. It is possible, for example, that the dynamics of spatial attention (see, e.g., the "zoom lens" model of Eriksen \& St. James, 1986) could depend on trial mixing, such that attention is more rapidly focused on the target in the blocked neutral condition or even pre-focused on only the target. If the

\footnotetext{
${ }^{3}$ While not part of the planned set of analyses, we did conduct tests of whether the apparent reversals of blocked/mixed effect near the end of the EMG and response-force waveforms were reliable. They were not. For EMG, the blocked/mixed effect during the interval from 300 to $400 \mathrm{~ms}$ after response onset was $-0.62 \pm 0.71 \mathrm{uV} ; \boldsymbol{t}(10)=0.87, p=.405$, adj $\widehat{\eta}_{\mathrm{p}}^{2}=-.023$. For response force, the effect in the same interval was $-23.29 \pm 13.78 \mathrm{cN}$; $\boldsymbol{t}(10)=1.69, p=.122, \operatorname{adj} \widehat{\eta}_{\mathrm{p}}^{2}=.144$.
} 
effects of this difference are allowed to feed forward to response preparation (due to continuous flow), then it could produce a blocked/mixed effect on the LRP. While this particular alternative would seem to provide a reasonable explanation of the original finding of a mixing effect on control trials (because control trials have no flankers and are visually quite distinct from congruent and incongruent trials), it is somewhat undermined by the identical finding of a mixing effect on neutral trials. However, it cannot be firmly rejected.

One might also question the value of testing an aspect of a model from more than 40 years ago. In response, first note that neither the finding of a blocked/mixed effect on neutral-trial performance nor the suggestion of trial-mixing-dependent response inhibition is often mentioned in papers that cite Eriksen's work. If one examines the ten papers that cite Eriksen and Eriksen (1974) and have, in turn, been cited the most (according to Web of Science in May of 2020), none mention either of these; they all focus on the flanker effect (i.e., the difference between congruent and incongruent trials), continuous flow, and response competition. Furthermore, one important review of the "costs" associated with mixing various trial-types within a block (Los, 1996) does not cite Eriksen's early work at all, nor does a recent review of how inhibition is often used as a form of cognitive control in order to avoid making errors (Braver, 2012). If nothing else, the present paper may help revive interest in some of the important but over-looked contributions of Charles W. Eriksen.

More critical, however, is how trial-mixing-dependent response inhibition is missing from many recent models of selective attention. For example, the well-known and widely cited model of Botvinick et al. (2001) does not include a mechanism that corresponds to the type of response inhibition that was proposed by Eriksen and Eriksen (1974) and supported by the present experiments. With a few notable exceptions (e.g., Sohn, Albert, Jung, Carter, \& Anderson, 2007), this type of "conflict-monitoring" model is only designed to explain the differences in processing that occur during the performance of congruent and incongruent trials; they cannot explain differences that are already in place before the trial begins, such as the blocked/mixed effect on control (or neutral) trials. In modern terms (see Braver, 2012), these models include reactive response inhibition, but not the pro-active response inhibition that Eriksen proposed and the present experiments supported.

Likewise, computational models of selective attention in general and the flanker task in particular (starting with the work of Cohen et al., 1992), including those based on diffusion processes with non-stationary drift (e.g., White et al., 2011), have not included pro-active response inhibition. The addition of such this well alter the answer to a central question: whether the transition between the non-selective and selective phases of processing is gradual or discrete all-or-none. So, yes, the Two-Phase Model of Charles W. Eriksen is old, but it contains several ideas that remain crucial today.
Acknowledgements The authors thank Cathleen Moore, Rose Halterman Danek, Gordon Logan, the reviewers, and the members of the HPP Group at the University of Iowa for their helpful comments.

\section{References}

Botvinick, M. M., Braver, T. S., Barch, D. M., Carter, C. S., \& Cohen, J. D. (2001). Conflict monitoring and cognitive control. Psychological Review, 108, 624-652.

Braver, T. S. (2012). The variable nature of cognitive control: A dualmechanisms framework. Trends in Cognitive Science, 16, 106-113.

Cohen, J. D., Servan-Schreiber, D., \& McClelland, J. L. (1992). A parallel distributed processing approach to automaticity. American Journal of Psychology, 105, 239-269.

Coles, M. G. H. (1989). Modern mind-brain reading: Psychophysiology, physiology, and cognition. Psychophysiology, 26, 251-269.

Coles, M. G. H., Gratton, G., Bashore, T. R., Eriksen, C. W., \& Donchin, E. (1985). A psychophysiological investigation of the continuous flow model of human information processing. Journal of Experimental Psychology: Human Perception and Performance, 11, 529-553.

Danek, R. H., \& Mordkoff, J. T. (2011). Unequal motor durations under simple-, go/no-go, and choice-RT tasks: extension of Miller and Low (2001). Journal of Experimental Psychology: Human Perception and Performance, 37, 1323-1329.

Donohue, S. E., Appelbaum, L. G., McKay, C. C., \& Woldorff, M. G. (2016). The neural dynamics of stimulus and response conflict processing as a function of response complexity and task demands. Neuropsychologia, 84, 14-28.

Eriksen, B. A. \& Eriksen, C. W. (1974). Effects of noise letters upon identification of a target letter in a nonsearch task. Perception \& Psychophysics, 16, 143-149.

Eriksen, C. W., \& Hoffman, J. E. (1972). Temporal and spatial characteristics of selective encoding from visual displays. Perception \& Psychophysics, 12, 201-204.

Eriksen, C. W., \& Hoffman, J. E. (1973). The extent of processing of noise elements during selective encoding from visual displays. Perception \& Psychophysics, 14, 155-160.

Eriksen, C. W., \& Schultz, D. W. (1979). Information processing in visual search: A continuous flow conception and experimental results. Perception \& Psychophysics, 25, 249-263.

Eriksen, C. W., \& St. James, J. D. (1986). Visual attention within and around the field of focal attention: A zoom lens model. Perception \& Psychophysics, 40, 225-240.

Giray, M., \& Ulrich, R. (1993). Motor coactivation revealed by response force in divided and focused attention. Journal of Experimental Psychology: Human Perception and Performance, 19, 1278-1291.

Gratton, G., Coles, M. G., \& Donchin, E. (1992). Optimizing the use of information: Strategic control of activation of responses. Journal of Experimental Psychology: General, 121, 480-506.

Gratton, G., Coles, M. G. H., Sirevaag, E. J., Eriksen, C. W., \& Donchin, E. (1988). Pre- and post-stimulus activation of response channels: A psychophysiological analysis. Journal of Experimental Psychology: Human Perception and Performance, 14, 331-344.

Hübner, R., Steinhauser, M., \& Lehle, C. (2010). A dual-stage two-phase model of selective attention. Psychological Review, 117, 759-784.

Lappin, J. S., \& Eriksen, C. W. (1966). Use of a delayed signal to stop a visual reaction-time response. Journal of Experimental Psychology, 72, 805-811.

Liu, Y. S., Holmes, P., \& Cohen, J. D. (2008). A neural network model of the Eriksen task: Reduction, analysis, and data fitting. Neural Computation, 20, 345-373. 
Logan, G. D., \& Cowan, W. B. (1984). On the ability to inhibit thought and action: A theory of an act of control. Psychological Review, 91, 295-327.

Los, S. A. (1996). On the origin of mixing costs: Exploring information processing in pure and mixed blocks of trials. Acta Psychologica, $95,145-188$.

Luck, S. J. (2005). An introduction to the event-related potential technique. Cambridge, MA: MIT Press.

Mordkoff, J. T. (2019). A simple method for removing bias from a popular measure of standardized effect size: Adjusted partial eta squared. Advances in Methods and Practices in Psychological Science, 2, 228-232.

Mordkoff, J. T., \& Grosjean, M. (2001). The lateralized readiness potential and response kinetics in response-time tasks. Psychophysiology, $38,777-786$

Okada, K. (2017). Negative estimate of variance-accounted-for effect size: How often it is obtained, and what happens if it is treated as zero. Behavior Research Methods, 49, 979-987.

Osman, A., Kornblum, S., \& Meyer, D. E. (1986). The point of no return in choice reaction time: Controlled and ballistic stages of response preparation. Journal of Experimental Psychology: Human Perception and Performance, 12, 243-258.

Rockstroh, B., Elbert, T., Canavan, A., Lutzenberger, W., \& Birbaumer, N. (1989). Slow cortical potentials and behavior, 2nd ed. Munich: Urban \& Schwarzenberg.

Sohn, M.-H., Albert, M. V., Jung, K., Carter, C. S., \& Anderson, J. R. (2007). Anticipation of conflict monitoring in the anterior cingulate cortex and the prefrontal cortex. Proceedings of the National Academy of Sciences (PNAS), 104, 10330-10334.

Tillman, C. M., \& Wiens, S. (2011). Behavioral and ERP indices of response conflict in Stroop and flanker tasks. Psychophysiology, 48, 1405-1411.

Verbruggen, F., \& Logan, G. D. (2008). Response inhibition in the stopsignal paradigm. Trends in Cognitive Science, 12, 418-424.

Wessel, J. R., \& Aron, A. R. (2017). On the globality of motor suppression: Unexpected events and their influence on behavior and cognition. Neuron, 93, 259-280.

White, C. N., Ratcliff, R., \& Starns, J. J. (2011). Diffusion models of the flanker task: Discrete versus gradual attentional selection. Cognitive Psychology, 63, 210-238.

Zhou, S., Xiong, S., Cheng, W., \& Wang, Y. (2019). Flanker paradigm contains conflict and distraction factors with distinct neural mechanisms: An ERP analysis in a 2-1 mapping task. Cognitive Neurodynamics, 13, 341-356.

None of the data or materials for the experiments reported here are available, and none of the experiments was preregistered.

Publisher's note Springer Nature remains neutral with regard to jurisdictional claims in published maps and institutional affiliations. 